\title{
Relation of Quality of Life, Activities and Participation with Contracture Categories, and Contracture Sites in Elderly Residents With Joint Contractures in Long-Term Care Facilities
}

\author{
Yi-chang Chen \\ National Taiwan University \\ Keh-chung Lin ( $\nabla$ tom610713@gmail.com ) \\ National Taiwan University \\ Shu-Hui Yeh \\ Mackay Medical College \\ Chih-Hung Wang \\ National Changhua University of Education \\ Ay-Woan Pan \\ National Taiwan University \\ Hao-Ling Chen \\ National Taiwan University \\ Chen-Jung Chen \\ Mackay Medical College
}

\section{Research Article}

Keywords: activity limitations, elderly residents, joint contractures, osteoarthritis, participation restrictions, quality of life

Posted Date: January 21st, 2021

DOI: https://doi.org/10.21203/rs.3.rs-144731/v1

License: (1) This work is licensed under a Creative Commons Attribution 4.0 International License. Read Full License 


\section{Abstract}

Background: Joint contractures and degenerative osteoarthritis are the most common joint diseases in the elderly, can lead to limited mobility in the elderly with diseases, can exacerbate symptoms, such as pain, stiffness and disability, and can interfere with social participation and quality of life, affecting mental health. However, relevant studies on this topic are very limited. The purpose of this study is to investigate the relationship of demographic characteristics and joint contracture categories and sites with the quality of life, activity limitations, and participation restrictions of elderly residents in long-term care facilities.

Methods: A cross-sectional observational study. Elderly individuals with joint contractures who were residents in long-term care facilities were recruited. The World Health Organization (WHO) Quality of Life and the WHO Disability Assessment Schedule 2.0 were used to survey the participants. Correlations, multiple linear regressions, and multiple analyses of variance, with joint contractures as the response variable, were used in the statistical analysis.

Results: The final statistical analysis included 232 participants. The explanatory power of contracture sites on activities and participation had a moderate strength of association $\left(\eta^{2}=.113\right)$. Compared with elderly residents with joint contractures and osteoarthritis in isolated upper limbs, elderly residents with joint contractures and osteoarthritis in both the upper and lower limbs had significantly worse activity and participation limitations. There were no significant differences in activity and participation between elderly residents with joint contractures affecting isolated upper limbs and elderly residents with joint contractures affecting isolated lower limbs $\left(F_{1,226}=2.604\right.$ and $F_{1,226}=.674$, n.s.). Osteoarthritis had the greatest impact on activity limitations and participation restrictions of elderly residents with joint contractures affecting both upper and lower limbs $\left(F_{1,226}=6.251, p=.014\right)$.

Conclusions: Elderly residents in long-term care facilities who are minorities and have non-mainstream religious beliefs, history of stroke and osteoarthritis are at high risk of developing activity limitations and participation restrictions. Moreover, compared with other contractile sites, regardless of osteoarthritis, elderly residents with joint contractures affecting both upper and lower limbs had the most substantial activity limitations and participation restrictions.

Trial registration: This study has been registered in the Chinese Clinical Trial Registry, Registration number and date: ChiCTR2000039889

\section{Implications For Rehabilitation}

- Although activity and participation involved many factors, they were found to be the most important domains for the quality of life of older long-term care facility residents.

- Joint contractures are a major cause of activity limitations and participation restrictions in elderly residents in long-term care facilities.

- Minority, non-mainstream religious beliefs, stroke, and osteoarthritis were the 4 key risk factors for activity limitations and participation restrictions of elderly residents with joint contractures at long-term care facilities.

- The framework of risk factors for activity limitations and participation restrictions that this study reports may be helpful for medical professionals and the implementation of interventions for older patients with contractures.

\section{Background}

The rapid increase in global life expectancy means that joint contractures and osteoarthritis (OA), the most common joint diseases in the elderly [1, 2], are becoming major global public health issues [3]. Joint contractures are present in more than

$20 \%$ of elderly residents in long-term care facilities, and more than $10 \%$ develop symptoms of OA [1]. OA ranks fifth among all forms of disability worldwide [4]. The annual direct medical expenditure due to joint contractures and OA is estimated to be as high as US $\$$ $56,575,384$ in England [5]. Claims data-based estimates consistently exceeded US $\$ 10,000$ for individuals in the United States [6]. OA is the most common joint disease in the elderly population, is a dynamic pathologic chronic degenerative joint disease with a multifactorial etiology, and usually involves the progressive structural destruction of key joints, the progressive loss of articular cartilage, subchondral bone sclerosis, cyst formation, the development of osteophytes, and concomitant local low-grade inflammation [7, 8]. Although the definition of joint contracture currently lacks consensus [9], joint contracture is generally believed to be the continuous loss of range of motion (ROM) caused by structural changes due to insufficient extension of muscles and tendons [10,11].

Joint ROM limitation is an important factor for the development of joint contractures in affected joints of many OA patients [12], and joint contractures have long been regarded as one of the features of OA $[13,14]$. Some scholars have proposed that the occurrence of joint contractures precedes OA. After OA develops, secondary capsular contractures may subsequently develop as complications, suggesting that

Page $2 / 20$ 
there may not be a causal relationship between the two [11, 14]. Because the pathologic features of the two are independent of each other, it is unlikely that the occurrence of one leads to the occurrence of the other (causal relationship). However, the two may be due to the same causes (for example, immobility), and there is a strong negative correlation between the occurrence site (regional) and prevalence of the two, indicating that there may be some indirect implications between the two that remain to be clarified [11]. This suggests that the effects of joint contractures or OA on activity and participation, which are considered to be the most important factors affecting the quality of life (QoL) of elderly residents in long-term care facilities, may vary; that is, the QoL, activity, and social participation of individuals with joint contractures or OA-affected joints may differ from those of individuals with other conditions [15, 16].

Joint contractures are present in many elderly residents in long-term care facilities [1]. Joint contractures resulted in limited and restricted joint ROM [1, 17], which exacerbates OA-related symptoms, such as immobility, pain, stiffness, and disability, and accelerates OA progression [18]. Elderly individuals may experience substantial constraints in mobility and thus may experience activity and participation restrictions, such as standing, walking, or socializing limitations [19-21], which, in turn, may affect the QoL of elderly residents in long-term care facilities $[8,22,23]$ and result in the disability of elderly residents and an increase in social expenditure [24]. In particular, elderly residents with joint contractures and OA have a higher chance of developing the abovementioned conditions than do elderly residents with OA only [18].

Joint contractures and OA have significant impacts on the activities and social participation of elderly residents in long-term care facilities [25]. For example, upper limb contractures may hinder independent eating, whereas lower limb contractures may restrict ambulation [17, 26]. Activity and participation are substantial factors related to the QoL of older residents in long-term care facilities. QoL, activities, and participation of the older population are important for study, but these factors remain relatively unexplored [16]. Few studies have focused on the effect of joint contractures and OA on QoL, activities, and social participation of the elderly.

To prevent spread of the coronavirus disease 2019 (COVID-19), elderly residents with joint contractures in long-term care facilities must also adhere to the lockdown measures, and the limitations placed on travel could further reduce the opportunities for activities and participation [27]. Joint contractures and OA may negatively affect the ability of elderly individuals to protect themselves (such as wearing gloves and masks, cleaning surfaces, and washing hands), which could increase their risk of contracting and dying of COVID-19. Marks [8] predicted that the morbidity and mortality of elderly individuals with joint contractures or OA in long-term care facilities could increase. Therefore, there is a reason to focus more on these issues as lockdowns and isolation can occur suddenly in any location during the COVID-19 pandemic. This study investigated the relationship of demographic characteristics and joint contracture category and sites of elderly residents in longterm care facilities with their QoL, activity limitations, and participation restrictions.

\section{Methods}

\section{Design}

This was a cross-sectional observational study.

\section{Setting and participants}

The study participants are residents of long-term care facilities. Based on regression power analysis, the ratio between the explanatory variables and the number of samples should be 1:15-30. Because there were 11 explanatory variables in this study, 161 to 330 participants with joint contractures were needed for study to achieve a sufficient level of statistical power [28]. The inclusion criteria were age $\geq 65$ years old, being a long-term care facility resident for $>6$ months, adequate language skills to complete or answer questionnaires, and at least one site of joint contracture. The exclusion criteria were cognitive dysfunction and major mental illnesses diagnosed by a neurologic examination.

\section{Measures}

\section{Disease-related and sociodemographic data}

Based on the recommendation by interRAl, an international collaborative project, sociodemographic data (e.g., sex and age) were recorded with the Minimum Data Set (MDS) tool [29]; the criteria for determining cognitive status, joint contracture site, and OA were also defined in this study. The cutoff score for cognitive dysfunction was < 25 points on the Mini-Mental State Examination (MMSE). The following outcomes are determined by Chinese Version of the Cognitive Abilities Screening Instrument (CASI C): illiterate (years of education = 0 , unable to read and write), < 50 points; some education (years of education = $1-5$ years, some reading and writing skills), < 68 points; and educated (years of education $=6$ years, able to read and write), $<80$ points [30]. The MMSE results were obtained from the medical records of the participants. Participants without MMSE results completed the CASI C. 
Joint contractures were confirmed by doctors, nurses, or therapists. The criterion for a contracture was a score of 3 on a 4-point scale (loss > 2/3 of joint ROM) [31]. In a supine or lateral position, passive ROM of 12 main joints of both upper limbs (shoulders, elbows and wrists) and both lower limbs (hips and knees) was measured using a goniometer and a 4-point scale [31]: $0=$ normal (no loss of joint ROM); $1=$ mild (loss $\leq 1 / 3$ of joint ROM); $2=$ moderate (loss $\leq 2 / 3$ of joint ROM); and $3=$ severe (loss $>2 / 3$ of joint ROM). The main advantage of the 4 point scale is that it can be used to measure ROM in a large number of joints within a limited time [31]. The interrater reliability of the 4-point scale was acceptable for the enrolled elderly residents with joint contractures in long-term care facilities, with a Kendall $\tau$ coefficient of 0.62 and bootstrapped $95 \%$ confidence interval (Cl) of 0.49-0.74 [32].

The criterion for determining OA was radiographic evidence. Residents with inflammatory arthropathies, such as seropositive arthropathies (rheumatoid arthritis or systemic lupus erythematosus), seronegative arthropathies (psoriatic arthritis or ankylosing spondylitis), crystal arthropathies (gout), and joint arthroplasty were excluded.

\section{The World Health Organization Quality of Life-BREF (WHOQOL-BREF)}

The Chinese version of the WHOQOL-BREF developed by WHOQOL research headquarters was used to evaluate the QoL of the participants. There are 26 items divided into 4 domains of physical health, psychological health, social relationships, and environment. Questionnaires with missing data exceeding $20 \%$ were discarded. Missing values were replaced by the average for the domain. If there were more than 2 missing values in a domain, the domain score was not calculated (except for domain 3 ; the score was calculated if there was $<1$ missing value). The Cronbach a (internal consistency) for the entire questionnaire was 0.90 , and the test-retest reliability for each domain was 0.75 . The Pearson correlation coefficient between each item and the relevant domain ranged from 0.45 to $0.82(p<.01)$, and the correlation among different domains was ranged from 0.48 to $0.63(p<.01)$. For the confirmatory factor analysis of construct validity, the structural equation model of the 4 domains echoed the designed potential structure of the questionnaire, and the comparative fitness index (CFI) of the 2 analyses was 0.886 , similar to that of the Hong Kong version $(\mathrm{CFI}=0.894)$ and similar to the result using global data $(\mathrm{CFI}=0.903)[33]$.

\section{WHO Disability Assessment Scale 2.0 (WHODAS 2.0)}

The 36 items of the World Health Organization (WHO) Disability Assessment Scale (WHODAS 2.0) were used to evaluate the activities and participation of the study participants, and the researchers used a 5-point Likert scale to determine the difficulties in the activities and participation. For scoring, the averages calculated according to the interpolation formula provided in the WHODAS 2.0 manual were used to replace missing data [34]. The score ranged from 0 (lowest difficulty) to 100 (maximum difficulty), and the score for each domain and the sum for the 6 domains were calculated. The higher the score was, the higher the degree of disability and the more severe the restriction. The severity of restrictions was determined based on the difficulty classification methods by the International Classification of Functioning, Disability and Health (ICF) and WHODAS 2.0. The classification of disability severity was as follows: below 4\%, no disability; 5-24\%, mild; $25-49 \%$, moderate; $50-95 \%$, severe; and greater than $96 \%$, extremely severe [35]. The 4 items related to job ability were removed because all of the participants were retired or unemployed, and the remaining 32 items were used. For the Chinese version of WHODAS 2.0, the Cronbach a (internal consistency), a reliability index, was 0.73 to 0.99 ; the intraclass correlation coefficient was 0.8 to 0.89 [36, 37 ]; the interrater reliability exceeded 0.7 ; the content and the concurrent validity of the validity index were partially related; the exploratory factor analysis (EFA) extracted 5 to 7 factors, and the explanatory powers were all higher than $55 \%$; and for confirmatory factor analysis, the factor loadings were all higher than 0.56 [36]. The Chinese version has excellent reliability and validity and is consistent with item response theory.

\section{Data collection procedures}

Health professionals who were not members of the research team underwent questionnaire and interview training and collected data using structured face-to-face interviews. Before each interview, the medical records of the participant were first examined to extract relevant socialdemographic data and diagnoses. Each participant completed the questionnaire independently. If a participant was unable to complete the questionnaire independently due to vision, hearing, or reading or writing problems, the researchers provided assistance, for example, explaining certain sentences to ensure that the meaning was clear. The assistance provided by the researchers was consistent across participants; for instance, the examples provided were the same. If a participant was unable to answer a specific item, then the participant was allowed to ask the best-known person on site (relatives or nursing staff). Questionnaires with $50 \%$ or more information provided by relatives or nursing staff were marked, and these questionnaires were not used in the data analysis.

Six residents from a nursing home in Taiwan conducted a pre-test of the questionnaire. To explore the feasibility and acceptability of the questionnaire, a blank space was left below each item to allow those participating in the pre-test to provide questions and suggestions when completing the questionnaire.

\section{Validity assurance and data analysis}

Page $4 / 20$ 
When statistical surveys are used, missing items, rejection by respondents, researcher negligence, and issues related to the questionnaire itself can result in outliers and missing data; therefore, linear interpolation is often used after a comparison with the original data. If $10 \%$ of the questionnaire comprised outliers and missing data, the questionnaire was excluded. Before the formal analysis, frequency analysis was first performed on each variable to minimize operational errors during data input. Descriptive statistics were used to characterize the study participants.

All participants were stratified according to contracture category (isolated contracture or contracture and OA) and contracture site (isolated to the upper limbs, isolated to the lower limbs, both the upper and lower limbs). The mean, standard deviation (SD), and eta correlation ratio ( $\eta$ ) were used for continuous variables (e.g., activities and participation and QoL). Absolute and relative frequencies and the Spearman rank order correlation coefficient $\left(r_{s}\right)$ were used for discrete variables, including ordinal variables, such as education and visiting frequency. The Pearson chi-squared $\left(\chi^{2}\right)$ was used for categorical variables (e.g., sex and marital status).

The Durbin-Watson statistical test was conducted to determine whether an autocorrelation existed between residuals. By using the "enter" method to conduct multiple linear regression analyses after dummy coding categorical variables, the possible associations between the response variables (contractures affecting participants in different groups) and explanatory variables (demographic data, QoL, and activities and participation) were further investigated. Only the significant variables in the regression analysis were included in the bivariate analysis [38]. The $95 \% \mathrm{Cl}$ was used, and the significance level of each statistical test was set as $p<.050$.

Multiple analysis of variance (MANOVA) was used to detect differences in QoL and activities and participation among subjects in different groups. Finally, the interaction effect between variables was tested. The explanation of a main effect of a significant variable must consider the interaction of 2 independent variables; therefore, the main effect was not analysed, but the simple main effect test was conducted to compare the difference in activities and participation between category and sites. SPSS 20.0 software (IBM Corp, Armonk, NY, USA) was used for data processing and analyses.

\section{Ethical considerations}

As specified by the Declaration of Helsinki, this study was approved by an institutional review board and was formally approved by the participating long-term care facilities. Before the study, the study process was explained in detail to the participants, and the study was performed only after written consent was obtained from each participant.

\section{Results}

Of the 27 long-term care facilities contacted, 12 agreed to participate in this study. The other 15 care facilities refused to participate for various reasons, including staff shortages, lack of interest, and difficulties regarding coordinating research projects. From these 12 care facilities, 432 elderly residents met the inclusion criteria; 298 were selected by simple random sampling, and 246 completed the questionnaire, for a response rate of $82.55 \%$. After 14 questionnaires with more than $10 \%$ missing data were excluded, 232 participants were included in the final statistical analysis. Figure 1 shows the detailed flow diagram for the study. The participants were an average age of $75.70 \pm 9.93$ years, and 56.5\% were men. Among the participants, $176(75.9 \%)$ had at least 1 isolated contracture, and $56(24.1 \%)$ had at least 1 site with both a contracture and OA. Joint contractures in 105 participants (45.3\%) were isolated and affected at least 1 lower limb, $82(35.3 \%)$ had joint contractures affecting both the upper and lower limbs, and 45 (19.4\%) had isolated joint contractures affecting at least 1 upper limb. The 3 most common comorbidities were hypertension (29.7\%), stroke (24.1\%), and cardiovascular diseases (23.3\%) (Table 1). 
Table 1

Demographic and Condition-Specific Characteristics of the Participants $(N=232)$

\begin{tabular}{|c|c|c|c|c|c|}
\hline Characteristics & Mean & & SD & & $\min -\max$ \\
\hline Age (years) & 75.703 & & 9.933 & & $65-98$ \\
\hline Length of residency (month) & 37.517 & & 43.479 & & $6-240$ \\
\hline Body mass index $\left(\mathrm{kg} / \mathrm{m}^{2}\right)$ & 22.320 & & 3.479 & & $12.11-34.67$ \\
\hline Characteristics & & No. & & $\%$ & \\
\hline \multicolumn{6}{|l|}{ Contracture category } \\
\hline Isolated contracture & & 176 & & 75.9 & \\
\hline Both the contracture and $\mathrm{OA}$ & & 56 & & 24.1 & \\
\hline \multicolumn{6}{|l|}{ Contracture sites } \\
\hline Isolated upper limbs & & 45 & & 19.4 & \\
\hline Isolated lower limbs & & 105 & & 45.3 & \\
\hline Both the upper and lower limbs & & 82 & & 35.3 & \\
\hline \multicolumn{6}{|l|}{ Sex } \\
\hline Female & & 101 & & 43.5 & \\
\hline Male & & 131 & & 56.5 & \\
\hline \multicolumn{6}{|l|}{ Ancestry/ethnicity } \\
\hline Min Nan & & 201 & & 86.6 & \\
\hline Hakka & & 8 & & 3.4 & \\
\hline Aborigines & & 3 & & 1.3 & \\
\hline Mainland Chinese & & 9 & & 3.9 & \\
\hline Others & & 11 & & 4.7 & \\
\hline \multicolumn{6}{|l|}{ Education } \\
\hline Primary and below & & 122 & & 52.6 & \\
\hline Junior high & & 49 & & 21.1 & \\
\hline Senior secondary & & 30 & & 12.9 & \\
\hline Higher & & 12 & & 5.2 & \\
\hline College/university and above & & 19 & & 8.2 & \\
\hline \multicolumn{6}{|l|}{ Marital status } \\
\hline Married & & 63 & & 27.2 & \\
\hline Widowed & & 82 & & 35.3 & \\
\hline Divorced & & 23 & & 9.9 & \\
\hline Single & & 64 & & 27.6 & \\
\hline \multicolumn{6}{|l|}{ Religion } \\
\hline Buddhism & & 52 & & 22.4 & \\
\hline Taoism & & 107 & & 46.1 & \\
\hline Christianity/Catholicism & & 52 & & 22.5 & \\
\hline
\end{tabular}




\begin{tabular}{|c|c|c|c|}
\hline Characteristics & Mean & SD & $\min -\max$ \\
\hline Atheist & 17 & & 7.3 \\
\hline Others & 4 & & 1.7 \\
\hline \multicolumn{4}{|c|}{ Visiting frequency (weeks) } \\
\hline $0-1$ & 163 & & 70.2 \\
\hline $2-3$ & 52 & & 22.4 \\
\hline $4-5$ & 6 & & 2.6 \\
\hline $6-7$ & 11 & & 4.7 \\
\hline \multicolumn{4}{|l|}{ Chronic diseases } \\
\hline Hypertension & 69 & & 29.7 \\
\hline Diabetes & 49 & & 21.1 \\
\hline Stroke & 56 & & 24.1 \\
\hline Cardiovascular disease & 54 & & 23.3 \\
\hline Cataract & 4 & & 1.7 \\
\hline Glaucoma & 1 & & .4 \\
\hline
\end{tabular}

\section{Correlations among demographic characteristics, QoL, activities and participation}

There was a significant correlation between contracture category and the following variables: age $(\eta=.176, p=.007)$, length of residency ( $\eta$ $=.123, p=.030)$, sex $\left(X^{2}=5.561, p=.018\right)$, religion $\left(X^{2}=14.656, p=.012\right)$, stroke $\left(X^{2}=14.219, p<.001\right)$, and cataract $\left(X^{2}=12.792, p<.001\right)$. The contingency coefficients for sex, religion, stroke, and cataract were $0.153,0.244,0.240$, and 0.229 , respectively, indicating that these 4 factors and the 2 contracture categories (isolated contracture and both contracture and $\mathrm{OA}$ ) had a significantly weak strength of association. There was a significant correlation between contracture site and the following variables:

ancestry/ethnicity $\left(X^{2}=24.066, p=.002\right)$, religion $\left(X^{2}=31.585, p<.001\right)$, diabetes $\left(X^{2}=6.082, p=.048\right)$, stroke $\left(X^{2}=14.020, p=.001\right)$, and activities and participation $(\eta=.277, p<.001)$. The contingency coefficients for ancestry/ethnicity, religion, diabetes, and stroke were 0.307 , $0.346,0.160$, and 0.239 , respectively, indicating that the 4 factors and the 3 types of contracture sites (isolated to the upper limbs, isolated to the lower limbs, and both upper and lower limbs) had a significantly weak strength of association (Table 2). 
Table 2

Correlation among demographic characteristics, quality of life, activities and participation $(N=232)$

\begin{tabular}{|c|c|c|c|c|c|c|c|c|}
\hline & Contrac & e cates & & & Contract & sites & & \\
\hline Variables & $\eta$ & $r_{s}$ & $\chi^{2}$ & $p$ & $\eta$ & $r_{s}$ & $x^{2}$ & $p$ \\
\hline Age (years) & $.176^{\star \star}$ & & & .007 & .086 & & & .381 \\
\hline Length of residency (months) & $.143^{\star}$ & & & .030 & .065 & & & .382 \\
\hline Body mass index & .006 & & & .924 & .123 & & & .326 \\
\hline Sex & & & $5.561^{\star}$ & .018 & & & 1.733 & .420 \\
\hline Ancestry/ethnicity & & & 4.055 & .399 & & & $24.066 * *$ & .002 \\
\hline Education & & .036 & & .581 & & -.010 & & .882 \\
\hline Marital status & & & .593 & .898 & & & 9.180 & .164 \\
\hline Religion & & & $14.656^{*}$ & .012 & & & $31.585^{\star \star \star}$ & $<.001$ \\
\hline Visiting frequency (weeks) & & .043 & & .510 & & & -.083 & .209 \\
\hline \multicolumn{9}{|l|}{ Chronic diseases } \\
\hline Hypertension & & & .505 & .220 & & & .418 & .811 \\
\hline Diabetes & & & 1.130 & .288 & & & $6.082^{*}$ & .048 \\
\hline Stroke & & & $14.219 \star \star \star$ & $<.001$ & & & $14.020 * *$ & .001 \\
\hline Cardiovascular disease & & & 3.250 & .071 & & & 2.095 & .351 \\
\hline Cataract & & & $12.792^{\star \star \star}$ & $<.001$ & & & 3.424 & .180 \\
\hline Glaucoma & & & .320 & .572 & & & 4.174 & .124 \\
\hline Activities and participation & .035 & & & .599 & $.277^{\star \star \star}$ & & & $<.001$ \\
\hline Quality of life & .078 & & & .235 & .101 & & & .134 \\
\hline
\end{tabular}

\section{Factors associated with activities and participation}

For the multiple linear regression analysis, only the significantly correlated variables (Table 2) were included. The Durbin-Watson statistic was 1.625, indicating that there was no remaining autocorrelation in residuals. The set of 17 main effects was significant $\left(F_{17,214}=6.916, p\right.$ $<.001)$, and for the proportion of the variance that can be explained by the activity and participation of elderly residents, the multiple determination coefficient $R^{2}$ was 0.355 (model 1 ), which was higher than the $R^{2}(>0.208)$ for the logistic regression [39, 40]. The condition index (condition number) was 34.239 , indicating that collinearity was reduced [28]. The following variables were significantly related to activities and participation: ancestry/ethnicity (other) $(\beta=-0.169, t=-2.802, p=.006)$, religion (Christianity/Catholicism) $(\beta=-0.246, t=-$ $3.675, p<.001)$, religion (other) $(\beta=0.268, t=4.405, p<.001)$, stroke (yes) $(\beta=0.270), t=4.456, p<.001)$, and contracture category (both contracture and $O A)(\beta=0.214, t=3.451, p=.001)$, indicating that these 5 factors were the most critical factors affecting activities and participation and collectively explain $29.8 \%$ of activities and participation (model 2 ).

Individual independent variables were further tested, and regression coefficients indicated that stroke had the best explanatory power (i.e., stroke can cause activity limitations and participation restrictions). The tolerance and variance inflation factor (VIF) statistics decreased to 0.922 and 1.085 , respectively, indicating that there was no significant collinearity (Table 3 ). 
Table 3

Multiple Linear regression analysis of related factors with activities and participation $(N=232)$

\begin{tabular}{|c|c|c|c|c|c|c|}
\hline \multirow[t]{2}{*}{ Factors } & \multirow[t]{2}{*}{ SE (B) } & \multirow[t]{2}{*}{ Beta ( $\beta)$} & \multirow[t]{2}{*}{$T$ value } & \multirow[t]{2}{*}{$p$} & \multicolumn{2}{|l|}{$95 \% \mathrm{Cl}$} \\
\hline & & & & & Lower & Upper \\
\hline (Constant) & 78.504 & & $4.130 * \star \star$ & $<.001$ & 41.035 & 115.973 \\
\hline Age (years) & .032 & .010 & .138 & .890 & -.575 & .362 \\
\hline Length of residency (months) & .025 & .032 & .549 & .584 & -.064 & .113 \\
\hline \multicolumn{7}{|l|}{ Sex } \\
\hline Male & 1.004 & .015 & .247 & .805 & -7.011 & 9.020 \\
\hline \multicolumn{7}{|l|}{ Ancestry/ethnicity } \\
\hline Hakka & .032 & .000 & .003 & .998 & -21.863 & 21.927 \\
\hline Aborigines & -6.296 & -.021 & -.369 & .712 & -39.924 & 27.331 \\
\hline Mainland Chinese & -17.841 & -.104 & -1.665 & .097 & -38.965 & 3.283 \\
\hline Other & -26.389 & -.169 & $-2.802^{\star \star}$ & .006 & -44.956 & -7.823 \\
\hline \multicolumn{7}{|l|}{ Religion } \\
\hline Buddhism & -6.191 & -.078 & -1.245 & .215 & -15.997 & 3.614 \\
\hline Christianity/Catholicism & -19.608 & -.246 & $-3.675^{\star \star \star}$ & $<.001$ & -30.125 & -9.092 \\
\hline Atheist & -8.007 & -.061 & -1.037 & .301 & -23.230 & 7.216 \\
\hline Others & 61.223 & .268 & $4.405^{\star \star \star}$ & $<.001$ & 33.828 & 88.618 \\
\hline \multicolumn{7}{|l|}{ Diabetes } \\
\hline No & -5.709 & -.070 & -1.208 & .228 & -15.027 & 3.608 \\
\hline \multicolumn{7}{|l|}{ Stroke } \\
\hline Yes & 20.988 & .270 & $4.456^{\star \star \star}$ & $<.001$ & 11.705 & 30.272 \\
\hline \multicolumn{7}{|l|}{ Cataract } \\
\hline Yes & -31.027 & -.122 & -1.901 & .059 & -63.200 & 1.145 \\
\hline \multicolumn{7}{|l|}{ Contracture category } \\
\hline Both the contracture and $\mathrm{OA}$ & 16.593 & .214 & $3.451^{\star *}$ & .001 & 7.117 & 26.069 \\
\hline \multicolumn{7}{|l|}{ Contracture sites } \\
\hline Isolated upper limbs & -9.920 & -.118 & -1.854 & .065 & -20.468 & .629 \\
\hline Both the upper and lower limbs & 5.575 & .080 & 1.238 & .217 & -3.303 & 14.453 \\
\hline Model 1 (17 total variables) & $R^{2}=.355$ & & $\operatorname{adj} R^{2}=.303$ & \multicolumn{3}{|c|}{$F(17,214)=6.916, p<.001$} \\
\hline Model 2 (5 significant variables) & $R^{2}=.298$ & & $\operatorname{adj} R^{2}=.282$ & \multicolumn{3}{|c|}{$F(5,226)=19.166, p<.001$} \\
\hline
\end{tabular}

\section{Comparison of the differences in QoL, activities and participation between} categories and sites

MANOVA was conducted to compare the differences between category and site with respect to QoL and activities and participation. For QoL $(F=2.117, p=.064)$ and activities and participation $(F=1.420, p=.218)$, the results for the Levene test of homogeneity of variance were not significant, indicating that there was no significant difference in the dispersion of samples between these 2 classifications (i.e., category and site). By contrast, for the elderly residents, statistically significant differences (Wilks $\lambda=0.882, F_{2,226}=14.444, p<.001$ ) were observed for activities and participation among the 3 contracture sites (isolated to the upper limbs, isolated to the lower limbs, and both the upper and 
lower limbs), indicating that residents with different contracture sites had significant differences in activities and participation. The post hoc Tukey honestly significant difference test for the 3 contracture sites and activities and participation indicated significant differences with respect to isolated to the upper limbs vs isolated to the lower limbs $(95 \% \mathrm{Cl},-28.109$ to $-1.511 ; p=.025)$ and isolated to the upper limbs vs. both the upper and lower limbs $(95 \% \mathrm{Cl},-39.674$ to $-11.980 ; p<.001)$.

The average activity and participation scores for patients with contractures isolated to the upper limbs (60.167) and isolated to the lower limbs (76.880) were significantly lower than that for both the upper and lower limbs (98.714), indicating that elderly residents with joint contractures affecting both the upper and lower limbs and contractures isolated to the lower limbs had more activity limitations and participation restrictions than did those with joint contractures isolated to the upper limbs. The explanatory power of contracture site for activities and participation was $11.3 \%$ in terms of the $\eta^{2}$ coefficient; therefore, the strength of association was moderate (Table 4). Contracture category and site affect activities and participation interactively; the profile plots (Figs. 2-5) clearly show an interaction effect and that the interaction effect was significant. Therefore, a test of the simple main effect was conducted to discuss under what circumstances activities and participation increase or decrease.

Table 4

MANOVA to compare the difference in quality of life, activities and participation between category and sites $(N=232)$

\begin{tabular}{|c|c|c|c|c|c|c|c|c|c|c|c|c|}
\hline \multirow{2}{*}{$\begin{array}{l}\text { Sources of } \\
\text { variation }\end{array}$} & \multicolumn{8}{|c|}{ Activities and participation } & \multicolumn{4}{|c|}{ Quality of life } \\
\hline & sS & $d f$ & MS & $F$ & $p$ & $\eta^{2}$ & sS & $d f$ & MS & $F$ & $p$ & $\eta^{2}$ \\
\hline $\begin{array}{l}\text { Contracture } \\
\text { category } \\
\text { (A) }\end{array}$ & 891.879 & 1 & 891.879 & .891 & .346 & .004 & 486.995 & 1 & 486.995 & 1.630 & .203 & .007 \\
\hline $\begin{array}{l}\text { Contracture } \\
\text { sites (B) }\end{array}$ & 28912.809 & 2 & 14456.404 & $14.444^{\star \star \star}$ & $<.001$ & .113 & 1040.411 & 2 & 520.205 & 1.741 & .178 & .015 \\
\hline$A \times B$ & 8826.055 & 2 & 4413.028 & $4.409 *$ & .013 & .038 & 632.126 & 2 & 316.063 & 1.058 & .349 & .009 \\
\hline Error & 226198.890 & 226 & 1000.880 & & & & 67525.910 & 226 & 298.787 & & & \\
\hline $\begin{array}{l}\text { Corrected } \\
\text { Total }\end{array}$ & 255908.106 & 231 & & & & & 69177.272 & 231 & & & & \\
\hline
\end{tabular}

\section{Simple main effect test to compare the difference in activities and participation between categories and sites}

Before testing for the main effect based on the analysis of variance (ANOVA), we used the family-wise error rate, $a_{F W}$, to avoid expansion of type I error. The $\mathrm{a}_{\mathrm{FW}}$ of each test was set at $\mathrm{a} / 5=.05 / 5=.01$ to control the overall type I error at the .05 level. For elderly residents with joint contractures affecting both the upper and lower limbs but without OA (i.e., isolated contracture), the average for activity limitations and participation restrictions was 85.652 , and for elderly residents with joint contractures and $\mathrm{OA}$, the average for activity limitations and participation restrictions was 111.775 , a result that was higher than that for residents with contractures isolated to the lower limbs. For those with contractures isolated to the upper limbs and with both joint contractures and $\mathrm{OA}$, the average for activity limitation and participation restriction was 51.923, and for those with contractures isolated to the upper limbs and without OA (i.e., isolated contractures), the average for activity limitation and participation restriction was 68.410 , but the increase was not significant.

The interaction effects of contracture category and contracture site on activities and participation were different in different groups. First, the effects of different contracture sites on the activities and participation of residents with joint contractures were analysed from the split file of 2 groups from the contracture category. In the isolated contractures group (i.e., without OA), the activities and participation of residents with joint contractures at different sites were significantly different $\left(F_{2,226}=3.311, p=.039\right)$. Post hoc test results found that activity limitations and participation restrictions of residents with joint contractures affecting both the upper and lower limbs $(M=85.652)$ were significantly higher than those of residents with joint contractures isolated to the upper limbs $(M=68.410)$ and were nonsignificantly higher than those of residents with joint contractures isolated to the lower limbs $(M=76.880)$. Pairwise comparisons showed that only the comparison between isolated to the upper limbs and both the upper and lower limbs was significant. In addition, in the contracture and OA group, the effect of contracture site on activity limitations and participation restrictions was not only significant but also increased $\left(F_{2,226}=13.799, p<.001\right)$. The post hoc test results indicated that the activity limitations and participation restrictions of residents with joint contractures affecting 
both the upper and lower limbs $(M=111.775)$ was significantly higher than those of residents with joint contractures isolated to the lower limbs $(M=82.223)$ and residents with joint contractures isolated to the upper limbs $(M=51.923)$; pairwise comparisons were all significant.

The effect of $\mathrm{OA}$ (i.e., contracture category) on the activities and participation of residents with joint contractures was analysed from the split file of 3 contracture site groups. In the participants with contracture isolated to the upper limbs and those isolated to the lower limbs, OA had no significant effect on the activities $\left(F_{1,226}=2.60\right)$ and participation $\left(F_{1,226}=.674\right)$ of residents with joint contractures $\left(F_{1,226}=2.604\right)$. However, in the participants with contractures in both the upper and lower limbs, OA had a significant effect on the activities and participation of residents with joint contractures $\left(F_{1,226}=6.251, p=.014\right)$ (Table 5). Because the simple main effect of contracture category involves only 2 levels, there was no need for a post hoc test, and the average scores of the 2 groups were compared.

Table 5

Simple main effect in ANOVA to compare the difference in activities and participation between category and sites $(N=232)$

\begin{tabular}{|c|c|c|c|c|c|c|}
\hline \multirow[t]{2}{*}{ Sources of variation } & \multicolumn{6}{|c|}{ Activities and participation } \\
\hline & SS & $d f$ & MS & $F$ & $p$ & Post hoc tests \\
\hline \multicolumn{7}{|l|}{ Contracture sites } \\
\hline Within isolated contracture & 7013.515 & 2 & 3506.758 & $3.311^{\star}$ & .039 & $C>B, C>A, B>A$ \\
\hline Within both the contracture and OA & 22387.676 & 2 & 1058.981 & $13.799 * \star \star$ & $<.001$ & $\mathrm{C}>\mathrm{B}, \mathrm{C}>\mathrm{A}, \mathrm{B}>\mathrm{A}$ \\
\hline \multicolumn{7}{|l|}{ Contracture category } \\
\hline Within isolated upper limbs (A) & 2512.912 & 1 & 2512.912 & 2.604 & .114 & \\
\hline Within isolated lower limbs (B) & 623.666 & 1 & 623.666 & .674 & .413 & \\
\hline Within both the upper and lower limbs (C) & 6990.654 & 1 & 6990.654 & $6.251^{\star}$ & .014 & \\
\hline Error & 226198.890 & 226 & 1000.880 & & & \\
\hline
\end{tabular}

\section{Discussion}

For contracture category, there were differences in age, length of residency, sex, religion, stroke, and cataract between residents with contractures with OA and those with contractures without OA. First, because there are 6 variables, some indirect implications may support the argument that there is no causal relationship between the contractures and OA [11]; namely, for joints with contractures and joints with $\mathrm{OA}$, the occurrence of the two (pathologic characteristics) may occur independently, and it is unlikely that the occurrence of one leads to the occurrence of the other (causal relationship). However, the two may be caused by the same factors (e.g., immobility) [41, 42] and are regularly associated [11].

Second, the results of this study indicated that there were significant differences in activities and participation between the 2 groups of elderly residents with joint contractures (i.e., elderly residents with and without OA affecting both the upper and lower limbs), meaning that compared with elderly residents without OA affecting both the upper and lower limbs, elderly residents with OA affecting both the upper and lower limbs had significantly more activity limitations and participation restrictions. This result is consistent with the findings by Campbell et al. [15]; that is, for elderly residents in long-term care facilities, the effects of joint contractures and OA on QoL, activities, and social participation could be different.

After a detailed analysis, this study further revealed differences in the 2 groups of elderly residents with joint contractures (i.e., residents with and without OA affecting both the upper and lower limbs). This study found that in terms of QoL, OA had almost no effect on the activity limitations and participation restrictions of residents with joint contractures, a result that is different from the findings by Campbell et al. [15]. There are 2 possible reasons for the difference. First, the Campbell et al. [15] study was a systematic review. In the review, the risk of bias in each research field was tested, blinding processes were conducted, and disagreements were settled by a third party. However, it is still difficult to avoid inherent bias, and there was a lack of information and uncertainty regarding the potential for bias.

Second, for studies included in the review, subjects were limited to patients aged older than 18 and receiving OA contracture treatment. However, in this study, we included subjects who were not receiving OA contracture treatment, and the participants were aged older than 65 years. The substantial differences in the design of these 2 studies likely contributed to the different results described above. An interesting finding is that there were no significant differences in the effect of contracture category (i.e., with and without OA) on QoL, activities, and 
social participation of residents; however, different contracture sites (i.e., isolated or both the upper and lower limbs) had significantly different effects on activities and social participation of residents. Future studies are needed to clarify the underlying mechanisms.

Finally, this study found that time factors (i.e., age and length of residency) had different influences on the risk of OA for the 2 groups of elderly residents with joint contractures in long-term care facilities. In other words, the older the resident was and the longer the individual was a resident in the facility, the higher the risk of OA, a result that indirectly echoes those from previous studies [12, 43]. Therefore, measures to prevent time factors associated with joint contractures and OA, such as avoiding prolonged joint immobility, are particularly important.

For contracture site, the results showed that there were differences in ancestry/ethnicity, religion, diabetes, stroke, activities, and participation among the 3 groups of elderly residents. First, the results from this study not only support the previous finding that joint contractures affecting both the upper and lower limbs have a major impact on activities and participation [17] but also indicate that there are significant differences in activity limitations and participation restrictions in elderly residents with joint contractures when comparing individuals with contractures isolated to the upper limbs vs. isolated to the lower limbs and individuals with contractures isolated to the upper limbs vs. affecting both the upper and lower limbs. However, there were no significant differences in activities and participation of elderly residents with joint contractures when comparing those with contractures isolated to the lower limbs vs. affecting both the upper and lower limbs.

Comparing the results of this study to the findings by Bartoszek et al. [20] that there are no significant differences in activities and participation of patients with joint contractures when comparing those with contractures isolated to the upper limbs, isolated to the lower limbs, or affecting both upper and lower limbs, there is a consistency and a difference. Regarding the consistency, the same level of restriction on activities and participation occurred in the 2 groups of elderly residents with joint contractures isolated to the lower limbs vs. affecting both the upper and lower limbs, and regarding the difference, varying degrees of restrictions on activities and participation occurred in the groups of elderly residents with joint contractures. The reason for the difference may be that the previous study did not perform active verification of the joint contracture diagnosis; therefore, validity was not absolutely certain, and this limitation may be the main cause for the final conclusion that there were no significant differences.

Second, this study found that the most critical factors affecting the activities and participation of elderly residents in long-term care facilities were ancestry/ethnicity (other), religion (Christianity/Catholicism), religion (other), stroke (yes), and contracture category (both contracture and $\mathrm{OA}$ ) and can collectively explain nearly one-third (28.2\%) of variance in activities and participation of elderly residents with joint contractures in long-term care facilities. Therefore, minority, non-mainstream religious beliefs, stroke, and OA were the 4 key risk factors for joint contractures in elderly individuals residing in long-term care facilities. In contrast, Christianity/Catholicism was a preventive factor.

Finally, the results showed that the explanatory power of contracture site for activities and participation had a moderate strength of association $\left(\eta^{2}=.113\right)$. Compared with other contractile sites, regardless of OA, residents with joint contractures affecting both the upper and lower limbs had the most substantial activity limitations and participation restrictions.

This study has resulted in 3 new discoveries. First, for the 3 groups of elderly residents with joint contractures and without OA (i.e., isolated to the upper limbs, isolated to the lower limbs, and both the upper and lower limbs), the difference in activities and participation between the elderly in only 2 groups (isolated to the upper limbs vs. both upper and lower limbs) was significantly different. In other words, compared with residents with joint contractures isolated to the upper limbs but without OA, elderly patients without OA but with joint contractures affecting both the upper and lower limbs had significantly more activity limitations and participation restrictions.

Second, for the 3 groups of elderly residents with joint contractures and with OA (i.e., isolated to the upper limbs, isolated to the lower limbs, and both the upper and lower limbs), pairwise comparisons showed there were significant differences in activities and participation among the groups. In other words, compared with the residents with OA and joint contractures isolated to the upper limbs, elderly patients with OA and with joint contractures affecting both the upper and lower limbs had significantly more activity limitations and participation restrictions. The same results were also found when comparing elderly residents with contractures isolated to the upper limbs vs. isolated to the lower limbs and those with contractures isolated to the lower limbs vs. affecting both the upper and lower limbs.

Finally, regardless of the contracture category or contracture site, stroke was an important key complication. A previous study also identified that contractures usually present together with stroke, raising the question of whether they are related [44-46].

Some potential limitations should be considered. First, although the sample size in this study satisfied the requirements for establishing stable person and item estimates and a power analysis, caution is needed when generalizing our results because of the small sample size.

Second, the ethnicity percentages do not represent the percentages in the general population, and there is the possibility of self-selection bias and omitted variables.

Page $12 / 20$ 
Finally, chronic diseases were assessed through elderly residents' self-reports and medical records. This approach may not be as rigorous as standardized diagnostic tests and may have inherent biases that may disadvantage certain groups, such as elderly residents who are unfamiliar with chronic diseases or who are not proficient at relating terms for chronic diseases.

\section{Conclusion}

The activities and participation of elderly residents in long-term care facilities varied when contracture sites were different. In addition, the explanatory power of contracture sites for activities and participation had a moderate strength of association. Compared with other contracture sites, regardless of $\mathrm{OA}$, residents with contracture sites on both the upper and lower limbs had the most activity limitations and participation restrictions. Elderly residents in long-term care facilities who were minorities and had non-mainstream religious beliefs, history of stroke, and OA were at high risk of developing activity limitations and participation restrictions. However, no significant association between joint contractures and QoL among elderly residents was found in our study.

The current study not only fills the gap in investigating activity and participation but also more comprehensively associates elderly residents with joint contractures in long-term care facilities, providing a basis for improving the activity and participation of this population. In addition, the results of this study can also be used to improve the care, rehabilitation, prevention, and research programs provided in long-term care facilities. For example, when formulating strategies to encourage the activity and participation of elderly residents with joint contractures, a summary of risk factors can be obtained; thus, objective and empirical evidence can be provided to support clinical practice and to build a more complete and comprehensive health network.

\section{List Of Abbreviations}

\section{CASI C}

Chinese Version of the Cognitive Abilities Screening Instrument; CFI:comparative fitness indices; Cl:confidence interval; COVID-19:coronavirus disease 2019; EFAs:exploratory factor analyses; ICF:International Classification of Functioning, Disability, and Health; MDS:minimum data set; MMSE:Mini-Mental Status Examination; OA:osteoarthritis; QoL:Quality of life; ROM:range of motion; MDS:Minimum Data Set; WHODAS 2.0:WHO Disability Assessment Schedule 2.0; WHOQOL-BREF:World Health Organization Quality of Life-BREF

\section{Declarations}

\section{Ethical approval and consent to participate}

This study was approved by the National Taiwan University Research Ethics Committee (No. 201905HM137), and official informed consent from each participant was obtained. No incentive was given to respondents.

\section{Consent for publication}

Not applicable.

\section{Availability of supporting data}

Data availability of this study has been provided in the Chinese Clinical Trial Registry, Registration number and date: ChiCTR2000039889. Hyperlinks: http://www.chictr.org.cn/listbycreater.aspx

\section{Funding}

This study was supported in part by the National Health Research Institutes (NHRI-EX105-10403PI, NHRI-EX106-10403PI, NHRIEX10610604PI, and NHRI-EX107-10403PI), the Ministry of Science and Technology (104-2314-B-002-019-MY3 and 107-2314-B-002-052), and the Mackay Medical College (RD1050178) in Taiwan.

\section{Availability of data and materials}

Datasets used and/or analysed during the current study will be made available from the corresponding author on reasonable request. Data on which the conclusions of the paper rely will be published in the manuscript.

\section{Authors' contributions}


YCC and KCL were the main investigators. YCC, KCL, SHY and AWP contributed to the study design, data curation, funding acquisition, resources and supervision of the study. SHY, AWP and HLC performed significantly to the investigation, methodology and project administration of the study. SHY, HLC and CJC helped perform the formal analysis, software, validation and visualization. YCC, KCL and AWP contributed to writing - original draft, review and editing. All authors read and approved the manuscript.

\section{Acknowledgements}

We would like to thank the elderly residents of the long-term care facilities who participated in the study.

\section{Competing interests}

The authors report no conflicts of interest.

\section{References}

1. Harrington C, Carrillo H, Garfield R, et al. Nursing facilities, staffing, residents and facility deficiencies, 2009 through 2016. San Francisco (CA): Kaiser Family Foundation; 2018.

2. Radstake TRDJ, Mclnnes IB, Bijlsma JWJ. World arthritis day 2018-perspectives on rheumatic musculoskeletal diseases. Eur J Immunol. 2018;48(10):1604-1606.

3. Yoon AP, Kane RL, Hutton DW, et al. Cost-effectiveness of recurrent dupuytren contracture treatment. JAMA Netw Open. 2020;3(10):e2019861.

4. Vos T, Flaxman AD, Naghavi M, et al. Years lived with disability (YLDs) for 1160 sequelae of 289 diseases and injuries 1990-2010: a systematic analysis for the global burden of disease study 2010. Lancet. 2012;380(9859):2163-2196.

5. Gerber RA, Perry R, Thompson R, et al. Dupuytren's contracture: a retrospective database analysis to assess clinical management and costs in England. BMC Musculoskelet Disord. 2011;12:73.

6. Xie F, Kovic B, Jin X, et al. Economic and humanistic burden of osteoarthritis: a systematic review of large sample studies. Pharmacoeconomics. 2016;34(11):1087-1100.

7. Charlesworth J, Fitzpatrick J, Perera NKP, et al. Osteoarthritis- a systematic review of long-term safety implications for osteoarthritis of the knee. BMC Musculoskelet Disord. 2019;20(1):151.

8. Marks R. Osteoarthritis in older adults: disability associations and the corona virus. Am J Aging Sci Res. 2020;1(1):1-6.

9. Gnass I, Bartoszek G, Thiesemann R, et al. Joint contractures in older age. A systematic literature review. Z Gerontol Geriatr. 2010;43(3):147-157.

10. Flint MH, Poole CA. Contraction and contracture. In: McFarlane RM, McGrouther DA, Flint MH, editors. Dupuytren's disease. Edinburgh: Churchill Livingstone; 1990. p. 104-116.

11. Jones P, Alexander CJ, Stewart J, et al. Idiopathic osteoarthritis and contracture: causal implications. Ann Rheum Dis. 2005;64(2):226228.

12. Campbell TM, Trudel G, Laneuville $O$. Knee flexion contractures in patients with osteoarthritis: clinical features and histologic characterization of the posterior capsule. PM R. 2015;7(5):466-473.

13. Alexander CJ. Idiopathic osteoarthritis: time to change paradigms? Skeletal Radiol. 2004;33(6):321-324.

14. O'Reilly S, Doherty M. Clinical features of osteoarthritis and standard approaches to the diagnosis. In: Brandt KD, Doherty M, Lohmander LS, editors. Osteoarthritis. Oxford: Oxford University Press; 1998. p. 197-217.

15. Campbell TM, Ghaedi BB, Ghogomu ET, et al. Effectiveness of stretching and bracing for the treatment of osteoarthritis-associated joint contractures prior to joint replacement: a systematic review protocol. BMJ Open. 2019;9(7):e028177.

16. Chen YC, Lin KC, Wu CY, et al. Determinants of quality of life in the older residents of long-term care facilities using the world health organization international classification of functioning, disability and health framework in Taiwan. Disabil Rehabil. 2020;42(16):23252333.

17. Fergusson D, Hutton B, Drodge A. The epidemiology of major joint contractures: a systematic review of the literature. Clin Orthop Relat Res. 2007;456:22-29.

18. Steultjens MP, Dekker J, Van Baar ME, et al. Range of joint motion and disability in patients with osteoarthritis of the knee or hip. Rheumatology (Oxford). 2000;39(9):955-961.

19. Fischer U, Müller M, Strobl R, et al. Examining functioning and contextual factors in individuals with joint contractures from the health professional perspective using the ICF: an international internet-based qualitative expert survey. Rehabil Nurs. 2016;41(3):170-178. 
20. Bartoszek G, Fischer U, Grill E, et al. Impact of joint contracture on older persons in a geriatric setting: a cross-sectional study. Z Gerontol Geriatr. 2015;48(7):625-632.

21. Saal S, Beutner K, Bogunski J, et al. Interventions for the prevention and treatment of disability due to acquired joint contractures in older people: a systematic review. Age Ageing. 2017;46(3):373-382.

22. Murphy MT, Skinner TL, Cresswell AG, et al. The effect of knee flexion contracture following total knee arthroplasty on the energy cost of walking. J Arthroplasty. 2014;29(1):85-89.

23. Portegijs E, Rantakokko M, Mikkola TM, et al. Association between physical performance and sense of autonomy in outdoor activities and life-space mobility in community-dwelling older people. J Am Geriatr Soc. 2014;62(4):615-621.

24. Hunter DJ, Bierma-Zeinstra S. Osteoarthritis. Lancet. 2019;393(10182):1745-1759.

25. Müller M, Oberhauser $\mathrm{C}$, Fischer $\mathrm{U}$, et al. The paarticular scales - a new outcome measure to quantify the impact of joint contractures on activities and participation in individuals in geriatric care: development and Rasch analysis. Int J Nurs Stud. 2016;59:107-117.

26. Harato K, Nagura T, Matsumoto $\mathrm{H}$, et al. Extension limitation in standing affects weight-bearing asymmetry after unilateral total knee arthroplasty. J Arthroplasty. 2010;25(2):225-229.

27. Corsi M, Alvarez C, Callahan LF, et al. Contributions of symptomatic osteoarthritis and physical function to incident cardiovascular disease. BMC Musculoskelet Disord. 2018;19(1):393.

28. Green SB. How many subjects does it take to do a regression analysis. Multivariate Behav Res. 1991;26(3):499-510.

29. InterRAI Country Websites. Long-Term Care Facilities (LTCF). 2006 [Cited 2020 Jan 22]. Available from: http: //www.interrai.org/longterm-care-facilities.html

30. Yang YH, Lai CL, Lin RT, et al. Cut-off values of blessed dementia rating scale and its clinical application in elderly Taiwanese. Kaohsiung J Med Sci. 2006;22(8):377-384.

31. Hoang PD, Gandevia SC, Herbert RD. Prevalence of joint contractures and muscle weakness in people with multiple sclerosis. Disabil Rehabil. 2014;36(19):1588-1593.

32. Kwah LK, Harvey LA, Diong JH, et al. Half of the adults who present to hospital with stroke develop at least one contracture within six months: an observational study. J Physiother. 2012;58(1):41-47.

33. Yao G, Wu CH, Yang CT. Examining the content validity of the WHOQOL-BREF from respondents' perspective by quantitative methods. Soc Indic Res. 2008;85(3):483-498.

34. Ustün TB, Chatterji S, Kostanjsek N, et al. Developing the world health organization disability assessment schedule 2.0. Bull World Health Organ. 2010;88(11):815-823.

35. Virués-Ortega J, De Pedro-Cuesta J, Seijo-Martínez M, et al. Prevalence of disability in a composite $\geq 75$ year-old population in Spain: a screening survey based on the international classification of functioning. BMC Public Health. 2011;11:176-187.

36. Chiu TY, Yen CF, Chou CH, et al. Development of traditional Chinese version of world health organization disability assessment schedule 2.0 36-item (WHODAS 2.0) in Taiwan: validity and reliability analyses. Res Dev Disabil. 2014;35(11):2812-2820.

37. Yen CF, Hwang AW, Liou TH, et al. Validity and reliability of the functioning disability evaluation scale-adult version based on the WHODAS 2.0-36 items. J Formos Med Assoc. 2014;113(11):839-849.

38. Olusegun AM, Dikko HG, Gulumbe SU. Identifying the limitation of stepwise selection for variable selection in regression analysis. Am J Theor Appl Stat. 2015;4(5):414-419.

39. McFadden D. Conditional logit analysis of qualitative choice behavior. In: Zarembka P, editor. Frontiers in Econometrics. Academic Press. 1973. pp. 105-142.

40. Pic, A. What's the Best R-Squared for Logistic Regression? Statisical horizons. 2013. Retrieved from https://statisticalhorizons.com/r2logistic

41. Fischer U, Bartoszek G, Müller M, et al. Patients' view on health-related aspects of functioning and disability of joint contractures: a qualitative interview study based on the international classification of functioning, disability and health (ICF). Disabil Rehabil. 2014;36(26):2225-2232.

42. Harrington C, Carrillo H, Dowdell M, et al. Nursing facilities, staffing, residents and facility deficiencies, 2005 through 2010 . San Francisco (CA): Department of Social and Behavioural Sciences, University of California; 2011.

43. Riddle DL, Kong X, Jiranek WA. Factors associated with rapid progression to knee arthroplasty: complete analysis of three-year data from the osteoarthritis initiative. Joint Bone Spine. 2012;79(3):298-303.

44. Ada L, O'Dwyer N, O'Neill E. Relation between spasticity, weakness and contracture of the elbow flexors and upper limb activity after stroke: an observational study. Disabil Rehabil. 2006;28(13-14):891-897. 
45. Sackley C, Brittle N, Patel S, et al. The prevalence of joint contractures, pressure sores, painful shoulder, other pain, falls, and depression in the year after a severely disabling stroke. Stroke. 2008;39(12):3329-3334.

46. Wagner LM, Capezuti E, Brush BL, et al. Contractures in frail nursing home residents. Geriatr Nurs. 2008;29(4):259-266.

\section{Figures}

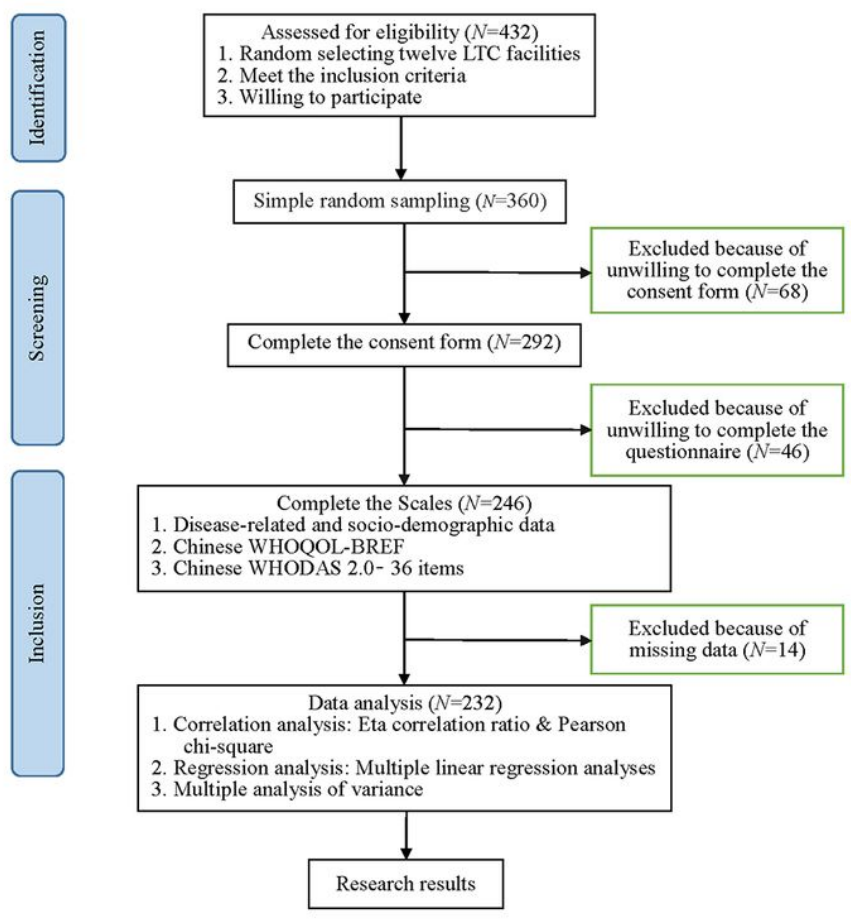

Figure 1

"Strengthening The Reporting of OBservational Studies in Epidemiology" (STROBE) diagram: flow of participants through the study. LTC: long-term care; WHOQOL-BREF: The World Health Organization Quality of Life-BREF; WHODAS 2.0: The World Health Organization Disability Assessment Schedule 2.0. 


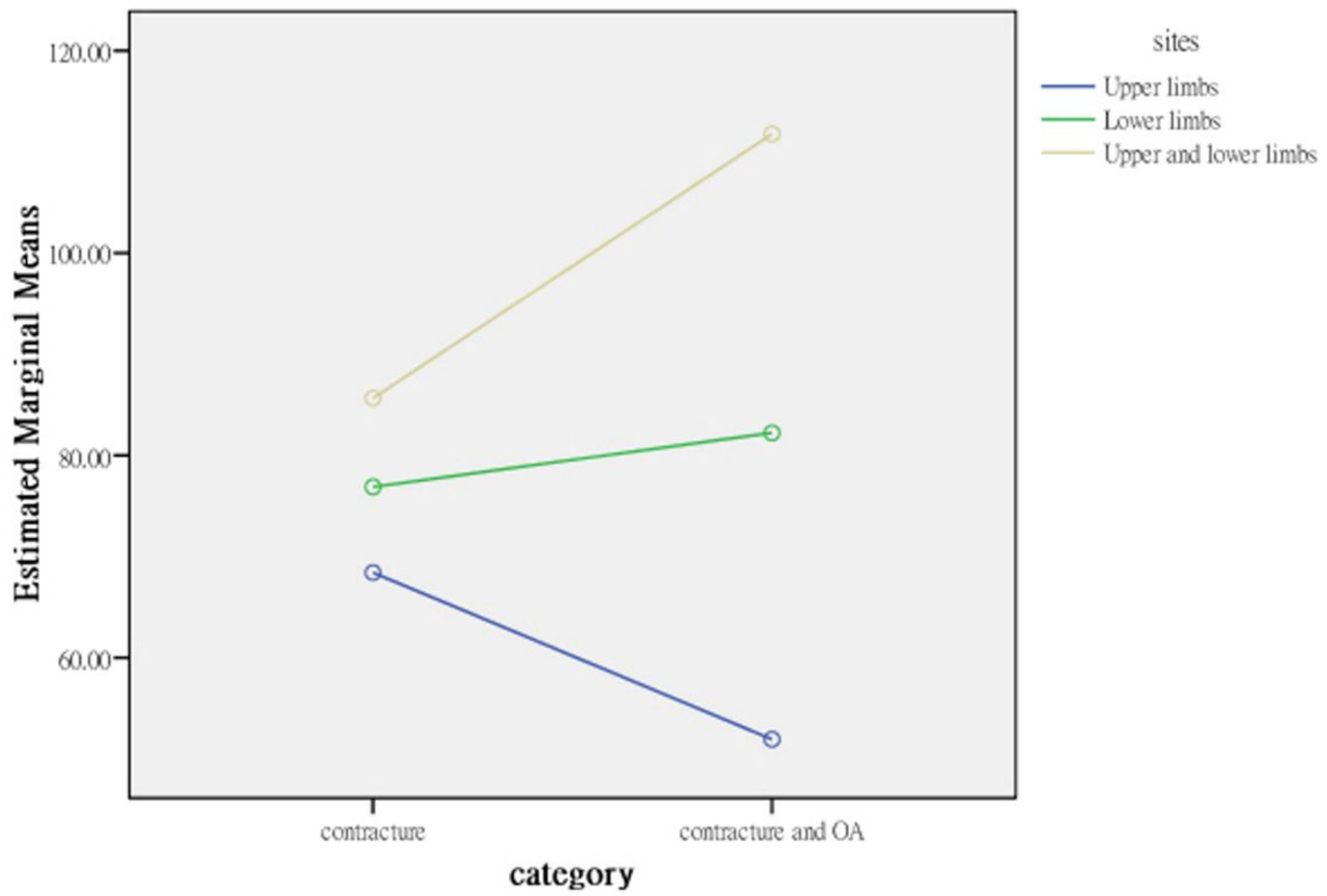

Figure 2

Interaction effect, activities and participation $\times$ contracture category. 


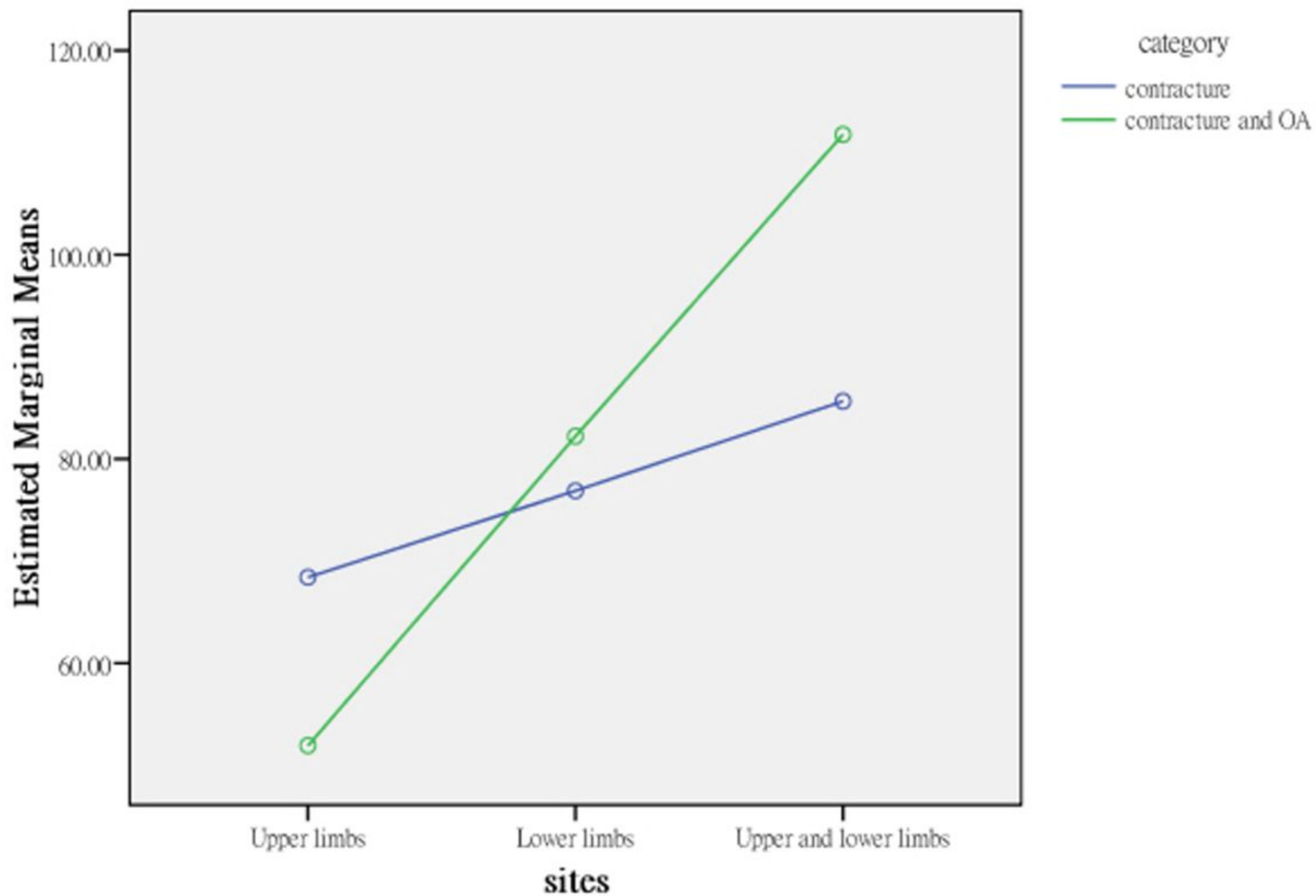

Figure 3

Interaction effect, activities and participation $\times$ contracture site. 


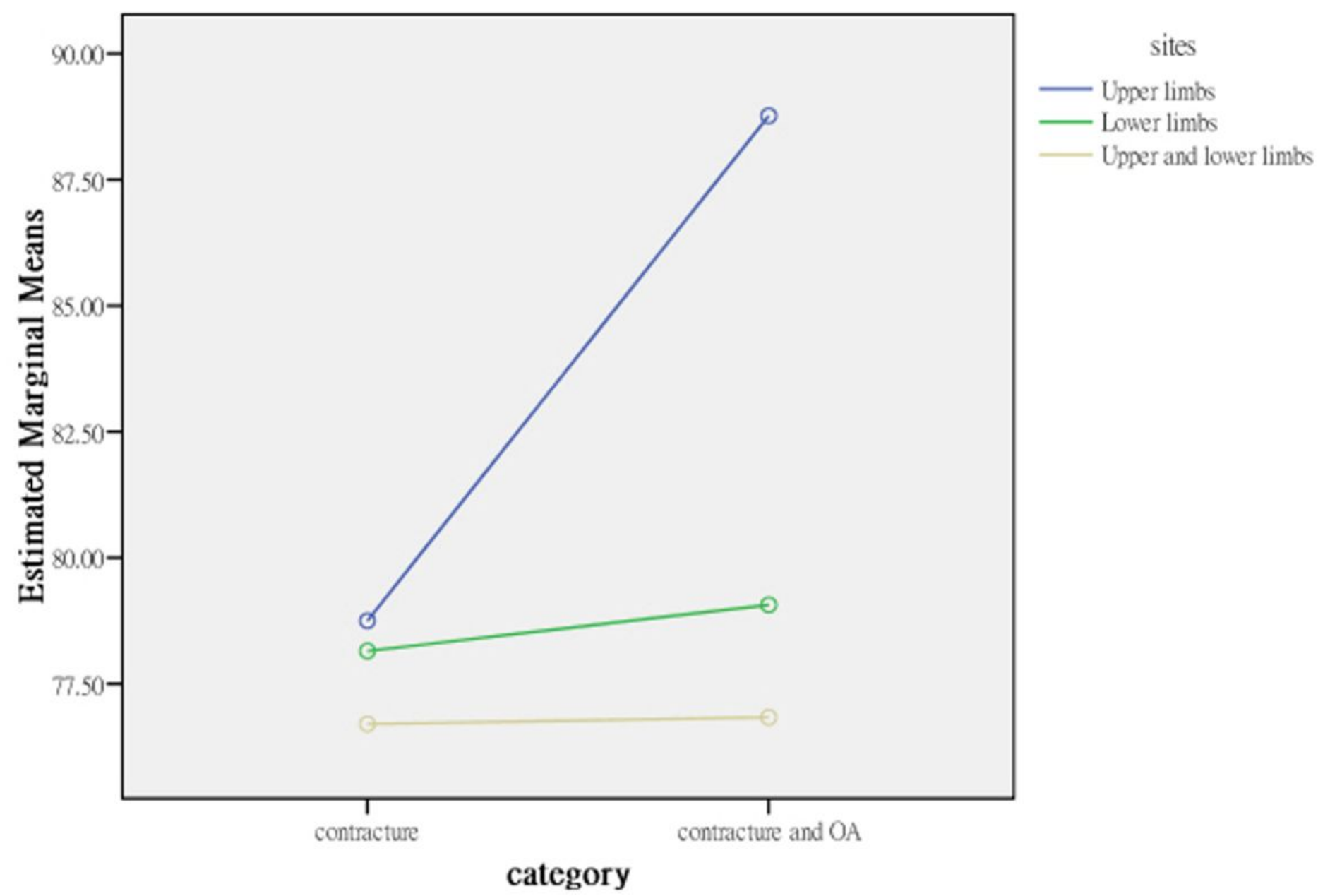

Figure 4

Interaction effect, quality of life $\times$ contracture category. 


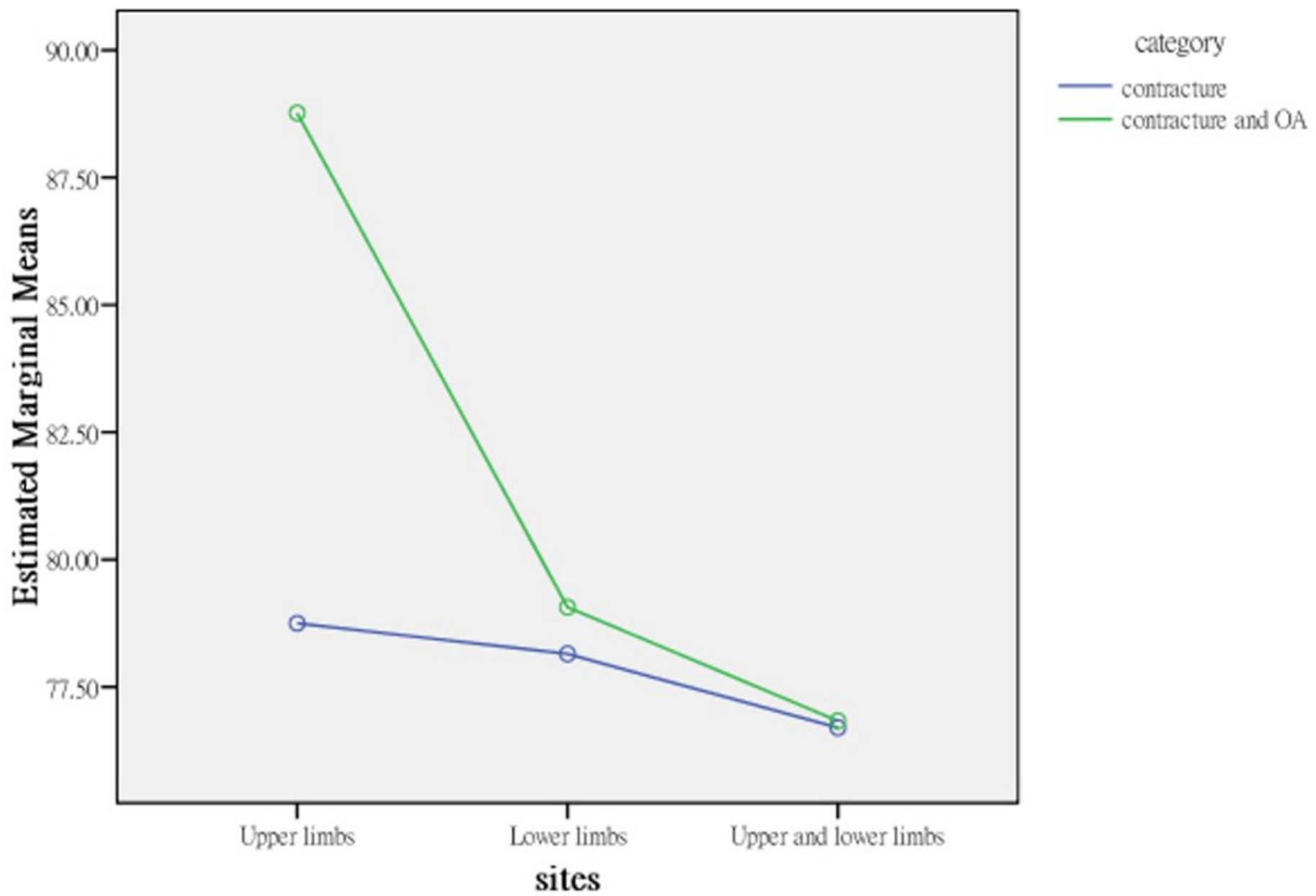

Figure 5

Interaction effect, quality of life $\times$ contracture sites. 